\title{
Relativistic Atomic Structure and Spectral Data Calculations for O-Like Tungsten Ion - Supplement
}

\author{
S.M. HAMASHA \\ Department of Physics, the Hashemite University, P.O. Box 150459, Zarqa, Jordan \\ Received: 20.01.2020 \& Accepted: 25.08.2020 \\ Doi: $10.12693 /$ APhysPolA.138.770.S1 \\ *e-mail: safeia@hu.edu.jo \\ topics: atomic data, plasma spectroscopy, transition probabilities, multipole transitions
}

TABLE SI

Possible electronic configurations of ground state complex $(n=2)$ and higher excited states $(n=3,4,5,6)$ in $\mathrm{W}^{66+}$

\begin{tabular}{c|l}
\hline \hline Complex & \multicolumn{1}{|c}{ Electronic configurations } \\
\hline$n=2$ & $2 s^{2} 2 p^{4}, 2 s^{1} 2 p^{5}, 2 p^{6}$ \\
\hline$n=3$ & $2 s^{2} 2 p^{3} 3 s, 2 s^{2} 2 p^{3} 3 p, 2 s^{2} 2 p^{3} 3 d, 2 s 2 p^{4} 3 s, 2 s 2 p^{4} 3 p, 2 s 2 p^{4} 3 d, 2 p^{5} 3 s, 2 p^{5} 3 p, 2 p^{5} 3 d$ \\
\hline \multirow{2}{*}{$n=4$} & $2 s^{2} 2 p^{3} 4 s, 2 s^{2} 2 p^{3} 4 p, 2 s^{2} 2 p^{3} 4 d, 2 s 2 p^{4} 4 s, 2 s 2 p^{4} 4 p, 2 s 2 p^{4} 4 d, 2 s 2 p^{4} 4 f, 2 s^{2} 2 p^{2} 4 s^{2}, 2 s^{2} 2 p^{2} 4 p^{2}, 2 s^{2} 2 p^{2} 4 d^{2}$, \\
& $2 s^{2} 2 p^{2} 4 f^{2}, 2 s^{2} 2 p^{1} 4 p^{3}, 2 s^{2} 2 p^{1} 4 d^{3}, 2 s^{2} 2 p^{1} 4 f^{3}, 2 s^{2} 4 p^{4}, 2 s^{2} 4 d^{4}, 2 s^{2} 4 f^{4}, 2 p^{5} 3 s, 2 p^{5} 4 p, 2 p^{5} 4 d, 2 p^{5} 3 f$ \\
\hline \multirow{5}{*}{$n=5$} & $2 s^{2} 2 p^{3} 5 s, 2 s^{2} 2 p^{3} 5 p, 2 s^{2} 2 p^{3} 5 d, 2 s^{2} 2 p^{3} 5 f, 2 s^{2} 2 p^{3} 5 g, 2 s 2 p^{4} 5 s, 2 s 2 p^{4} 5 p, 2 s 2 p^{4} 5 d, 2 s 2 p^{4} 5 f, 2 s 2 p^{4} 5 g$, \\
& $2 s^{2} 2 p^{2} 5 s^{2}, 2 s^{2} 2 p^{2} 5 p^{2}, 2 s^{2} 2 p^{2} 5 d^{2}, 2 s^{2} 2 p^{2} 5 f^{2}, 2 s^{2} 2 p^{2} 5 g^{2}, 2 s^{2} 2 p^{1} 5 p^{3}, 2 s^{2} 2 p^{1} 5 d^{3}, 2 s^{2} 2 p^{1} 5 f^{3}, 2 s^{2} 2 p^{1} 5 g^{3}$, \\
& $2 s^{2} 5 p^{4}, 2 s^{2} 5 d^{4}, 2 s^{2} 5 f^{4}, 2 s^{2} 5 g^{4}, 2 s 5 p^{5}, 2 s 5 d^{5}, 2 s 5 f^{5}, 2 s 5 g^{5}, 2 p^{5} 5 s, 2 p^{5} 5 p, 2 p^{5} 5 d, 2 p^{5} 5 f, 2 p^{5} 5 g$ \\
\hline \multirow{5}{*}{$n=6$} & $2 s^{2} 2 p^{3} 6 s, 2 s^{2} 2 p^{3} 6 p, 2 s^{2} 2 p^{3} 6 d, 2 s^{2} 2 p^{3} 6 f, 2 s^{2} 2 p^{3} 6 g, 2 s^{2} 2 p^{3} 6 h, 2 s 2 p^{4} 6 s, 2 s 2 p^{4} 6 p, 2 s 2 p^{4} 6 d, 2 s 2 p^{4} 6 f$, \\
& $2 s 2 p^{4} 6 g, 2 s 2 p^{4} 6 h, 2 s^{2} 2 p^{2} 6 s^{2}, 2 s^{2} 2 p^{2} 6 p^{2}, 2 s^{2} 2 p^{2} 6 d^{2}, 2 s^{2} 2 p^{2} 6 f^{2}, 2 s^{2} 2 p^{2} 6 g^{2}, 2 s^{2} 2 p^{2} 6 h^{2}, 2 s^{2} 2 p^{1} 6 p^{3}$, \\
& $2 s^{2} 2 p^{1} 6 d^{3}, 2 s^{2} 2 p^{1} 6 f^{3}, 2 s^{2} 2 p^{1} 6 g^{3}, 2 s^{2} 2 p^{1} 6 h^{3}, 2 s^{2} 6 p^{4}, 2 s^{2} 6 d^{4}, 2 s^{2} 6 f^{4}, 2 s^{2} 6 g^{4}, 2 s^{2} 6 g^{4}, 2 s 6 p^{5}, 2 s 6 d^{5}$, \\
& $2 s 6 f^{5}, 2 s 6 g^{5}, 2 s 6 h^{5}, 2 p^{5} 6 s, 2 p^{5} 6 p, 2 p^{5} 6 d, 2 p^{5} 6 f, 2 p^{5} 6 g, 2 p^{5} 6 h$
\end{tabular}

Strong electric dipole transitions (E1) atomic data for $\mathrm{W}^{66+}$ calculated by MR-MBPT method.

TABLE SII

\begin{tabular}{l|c|l|c|c|c|c|c}
\hline \hline \multicolumn{1}{c|}{ Upper state } & $J_{\text {up }}$ & Lower state & $J_{\text {low }}$ & $\Delta E[\mathrm{eV}]$ & $g f_{i j}$ & $A_{r}\left[\mathrm{~s}^{-1}\right]$ & $\lambda[\AA]$ \\
\hline $2 p_{1 / 2} 2 p_{3 / 2}^{2} 6 d_{3 / 2}$ & 1 & $2 p_{3 / 2}^{2}$ & 0 & 15964.19 & $4.78 \times 10^{-2}$ & $1.76 \times 10^{+14}$ & 0.7766 \\
$2 s_{1 / 2} 2 p_{1 / 2} 2 p_{3 / 2}^{3} 5 d_{5 / 2}$ & 1 & $2 s_{1 / 2} 2 p_{1 / 2}$ & 1 & 15199.64 & $1.18 \times 10^{-1}$ & $3.23 \times 10^{+14}$ & 0.8157 \\
$2 p_{1 / 2} 2 p_{3 / 2}^{2} 5 d_{3 / 2}$ & 1 & $2 p_{3 / 2}^{2}$ & 0 & 15177.62 & $9.72 \times 10^{-2}$ & $3.25 \times 10^{+14}$ & 0.8169 \\
$2 s_{1 / 2} 2 p_{3 / 2}^{2} 4 p_{3 / 2}$ & 1 & $2 p_{3 / 2}^{2}$ & 0 & 14108.07 & $8.61 \times 10^{-2}$ & $2.48 \times 10^{+14}$ & 0.8788 \\
$2 s_{1 / 2} 2 p_{1 / 2} 2 p_{3 / 2}^{3} 4 p_{3 / 2}$ & 1 & $2 p_{1 / 2} 2 p_{3 / 2}^{3}$ & 2 & 14105.83 & $8.55 \times 10^{-2}$ & $2.46 \times 10^{+14}$ & 0.8789 \\
$2 s_{1 / 2} 2 p_{3 / 2}^{2} 4 p_{3 / 2}$ & 1 & $2 p_{3 / 2}^{2}$ & 2 & 14062.58 & $8.18 \times 10^{-2}$ & $2.34 \times 10^{+14}$ & 0.8816 \\
$2 s_{1 / 2} 2 p_{1 / 2} 2 p_{3 / 2}^{3}$ & 1 & $2 s_{1 / 2} 2 p_{3 / 2}^{3}$ & 1 & 13807.12 & $4.28 \times 10^{-1}$ & $7.06 \times 10^{+14}$ & 0.8979 \\
$2 s_{1 / 2} 2 p_{3 / 2}^{2} 3 p_{1 / 2}$ & 1 & $2 p_{3 / 2}^{2}$ & 0 & 13782.22 & $3.46 \times 10^{-1}$ & $5.57 \times 10^{+14}$ & 0.8996 \\
$2 p_{1 / 2} 2 p_{3 / 2}^{2} 4 d_{3 / 2}$ & 1 & $2 p_{3 / 2}^{2}$ & 2 & 13760.43 & $2.38 \times 10^{-1}$ & $6.53 \times 10^{+14}$ & 0.901 \\
$2 p_{1 / 2} 4 d_{3 / 2}$ & 1 & $2 p_{3 / 2}^{4}$ & 0 & 13759.19 & $2.47 \times 10^{-1}$ & $6.72 \times 10^{+14}$ & 0.9011 \\
$2 s_{1 / 2} 2 p_{1 / 2} 2 p_{3 / 2}^{3} 4 d_{3 / 2}$ & 0 & $2 s_{1 / 2} 2 p_{3 / 2}^{3}$ & 1 & 13751.31 & $8.09 \times 10^{-2}$ & $6.64 \times 10^{+14}$ & 0.9016 \\
$2 s_{1 / 2} 2 p_{3 / 2}^{2} 3 p_{1 / 2}$ & 1 & $2 p_{3 / 2}^{2}$ & 2 & 12426.08 & $3.86 \times 10^{-1}$ & $6.29 \times 10^{+14}$ & 0.9977 \\
$2 s_{1 / 2} 2 p_{1 / 2} 2 p_{3 / 2}^{3}$ & 1 & $2 s_{1 / 2} 2 p_{1 / 2}$ & 0 & 12419.41 & $8.19 \times 10^{-1}$ & $1.04 \times 10^{+15}$ & 0.9983 \\
$2 p_{3 / 2}^{3} 3 d_{3 / 2}$ & 0 & $2 p_{1 / 2} 2 p_{3 / 2}^{3}$ & 1 & 12413.97 & $2.62 \times 10^{-1}$ & $1.31 \times 10^{+15}$ & 0.9987
\end{tabular}


TABLE SII cont.

\begin{tabular}{|c|c|c|c|c|c|c|c|}
\hline Upper state & $J_{\text {up }}$ & Lower state & $J_{\text {low }}$ & $\Delta E[\mathrm{eV}]$ & $g f_{i j}$ & $A_{r}\left[\mathrm{~s}^{-1}\right]$ & $\lambda[\AA]$ \\
\hline $2 p_{3 / 2}^{3} 3 d_{3 / 2}$ & 1 & $2 p_{1 / 2} 2 p_{3 / 2}^{3}$ & 1 & 12412.85 & $6.50 \times 10^{-1}$ & $1.09 \times 10^{+15}$ & 0.9988 \\
\hline $2 p_{3 / 2}^{3} 4 d_{3 / 2}$ & 3 & $2 p_{1 / 2} 2 p_{3 / 2}^{3}$ & 2 & 12353.66 & $4.44 \times 10^{-1}$ & $5.25 \times 10^{+14}$ & 1.0036 \\
\hline $2 p_{3 / 2}^{3} 4 d_{5 / 2}$ & 1 & $2 p_{3 / 2}^{4}$ & 0 & 12338.45 & $4.66 \times 10^{-1}$ & $1.04 \times 10^{+15}$ & 1.0048 \\
\hline $2 p_{1 / 2} 2 p_{3 / 2}^{2} 4 d_{5 / 2}$ & 2 & $2 p_{1 / 2} 2 p_{3 / 2}^{3}$ & 1 & 11015.47 & $4.55 \times 10^{-1}$ & $6.08 \times 10^{+14}$ & 1.1255 \\
\hline $2 s_{1 / 2} 3 p_{3 / 2}$ & 1 & $2 p_{3 / 2}^{4}$ & 0 & 10947.8 & $2.87 \times 10^{-1}$ & $5.01 \times 10^{+14}$ & 1.1325 \\
\hline $2 s_{1 / 2} 2 p_{1 / 2} 2 p_{3 / 2}^{3} 4 d_{3 / 2}$ & 1 & $2 s_{1 / 2} 2 p_{3 / 2}^{3}$ & 2 & 10903.64 & $2.47 \times 10^{-1}$ & $6.77 \times 10^{+14}$ & 1.1371 \\
\hline $2 p_{3 / 2}^{3} 3 p_{1 / 2}$ & 1 & $2 s_{1 / 2} 2 p_{3 / 2}^{3}$ & 2 & 10751.29 & $6.48 \times 10^{-1}$ & $1.06 \times 10^{+15}$ & 1.1532 \\
\hline $2 p_{1 / 2} 2 p_{3 / 2}^{2} 6 f_{9 / 2}$ & 1 & $2 p_{3 / 2}^{4}$ & 0 & 10746.4 & $4.68 \times 10^{-1}$ & $1.03 \times 10^{+15}$ & 1.1537 \\
\hline $2 s_{1 / 2} 2 p_{1 / 2} 2 p_{3 / 2}^{3}$ & 1 & $2 s_{1 / 2} 2 p_{3 / 2}^{3}$ & 1 & 10741.89 & $6.67 \times 10^{-1}$ & $1.11 \times 10^{+15}$ & 1.1542 \\
\hline $2 s_{1 / 2} 2 p_{1 / 2} 2 p_{3 / 2}^{3}$ & 3 & $2 s_{1 / 2} 2 p_{3 / 2}^{3}$ & 2 & 10733.22 & $1.76 \times 10^{0}$ & $1.25 \times 10^{+15}$ & 1.1551 \\
\hline $2 s_{1 / 2} 3 d_{3 / 2}$ & 2 & $2 s_{1 / 2} 2 p_{1 / 2}$ & 1 & 10715.51 & $1.45 \times 10^{0}$ & $1.45 \times 10^{+15}$ & 1.157 \\
\hline $2 p_{3 / 2}^{3} 3 d_{3 / 2}$ & 3 & $2 p_{1 / 2} 2 p_{3 / 2}^{3}$ & 2 & 10712.94 & $1.91 \times 10^{0}$ & $1.37 \times 10^{+15}$ & 1.1573 \\
\hline $2 p_{1 / 2} 2 p_{3 / 2}^{2} 3 d_{3 / 2}$ & 1 & $2 p_{3 / 2}^{2}$ & 2 & 10712.1 & $9.45 \times 10^{-1}$ & $1.57 \times 10^{+15}$ & 1.1574 \\
\hline $2 s_{1 / 2} 2 p_{1 / 2} 2 p_{3 / 2}^{3}$ & 2 & $2 s_{1 / 2} 2 p_{3 / 2}^{3}$ & 2 & 10711.46 & $1.44 \times 10^{0}$ & $1.43 \times 10^{+15}$ & 1.1575 \\
\hline $2 s_{1 / 2} 2 p_{1 / 2} 2 p_{3 / 2}^{3}$ & 1 & $2 s_{1 / 2} 2 p_{1 / 2}$ & 1 & 10695.96 & $1.43 \times 10^{0}$ & $1.81 \times 10^{+15}$ & 1.1591 \\
\hline $2 s_{1 / 2} 2 p_{1 / 2} 2 p_{3 / 2}^{3}$ & 2 & $2 s_{1 / 2} 2 p_{3 / 2}^{3}$ & 1 & 10691.64 & $1.59 \times 10^{0}$ & $1.57 \times 10^{+15}$ & 1.1596 \\
\hline $2 p_{1 / 2} 2 p_{3 / 2}^{2} 3 d_{3 / 2}$ & 2 & $2 p_{3 / 2}^{2}$ & 2 & 10683.84 & $1.53 \times 10^{0}$ & $1.53 \times 10^{+15}$ & 1.1604 \\
\hline $2 s_{1 / 2} 2 p_{1 / 2} 2 p_{3 / 2}^{3}$ & 0 & $2 s_{1 / 2} 2 p_{3 / 2}^{3}$ & 1 & 10681.94 & $3.61 \times 10^{-1}$ & $1.79 \times 10^{+15}$ & 1.1607 \\
\hline $2 s_{1 / 2} 2 p_{1 / 2} 2 p_{3 / 2}^{3}$ & 2 & $2 p_{1 / 2} 2 p_{3 / 2}^{3}$ & 1 & 10658.73 & $1.60 \times 10^{-1}$ & $1.65 \times 10^{+14}$ & 1.1632 \\
\hline $2 p_{3 / 2} 3 d_{5 / 2}$ & 1 & $2 p_{3 / 2}^{2}$ & 0 & 10649.85 & $1.14 \times 10^{0}$ & $1.45 \times 10^{+15}$ & 1.1641 \\
\hline $2 p_{1 / 2} 2 p_{3 / 2}^{2} 3 d_{5 / 2}$ & 0 & $2 p_{1 / 2} 2 p_{3 / 2}^{3}$ & 1 & 10621.51 & $2.10 \times 10^{-1}$ & $8.05 \times 10^{+14}$ & 1.1673 \\
\hline $2 s_{1 / 2} 2 p_{1 / 2} 2 p_{3 / 2}^{3}$ & 1 & $2 s_{1 / 2} 2 p_{1 / 2}$ & 0 & 10612.68 & $5.94 \times 10^{-1}$ & $7.51 \times 10^{+14}$ & 1.1682 \\
\hline $2 s_{1 / 2} 2 p_{1 / 2} 2 p_{3 / 2}^{3}$ & 1 & $2 s_{1 / 2} 2 p_{1 / 2}$ & 0 & 10504.75 & $7.31 \times 10^{-1}$ & $9.46 \times 10^{+14}$ & 1.1802 \\
\hline $2 s_{1 / 2} 2 p_{3 / 2}^{2} 3 d_{5 / 2}$ & 1 & $2 s_{1 / 2} 2 p_{3 / 2}^{3}$ & 1 & 9457.2 & $1.28 \times 10^{0}$ & $1.62 \times 10^{+15}$ & 1.311 \\
\hline $2 p_{1 / 2} 2 p_{3 / 2}^{2} 3 d_{3 / 2}$ & 3 & $2 p_{3 / 2}^{2}$ & 2 & 9442.09 & $2.13 \times 10^{0}$ & $1.52 \times 10^{+15}$ & 1.3131 \\
\hline $2 p_{3 / 2}^{3} 3 d_{5 / 2}$ & 1 & $2 p_{3 / 2}^{4}$ & 0 & 9435.53 & $2.38 \times 10^{0}$ & $3.01 \times 10^{+15}$ & 1.314 \\
\hline $2 s_{1 / 2} 2 p_{3 / 2}^{2} 3 d_{5 / 2}$ & 2 & $2 s_{1 / 2} 2 p_{3 / 2}^{3}$ & 2 & 9435.41 & $2.44 \times 10^{0}$ & $1.86 \times 10^{+15}$ & 1.314 \\
\hline $2 s_{1 / 2} 2 p_{1 / 2} 2 p_{3 / 2}^{3}$ & 1 & $2 s_{1 / 2} 2 p_{1 / 2}$ & 1 & 9424.98 & $9.70 \times 10^{-1}$ & $1.24 \times 10^{+15}$ & 1.3154 \\
\hline $2 p_{1 / 2} 2 p_{3 / 2}^{2} 3 d_{5 / 2}$ & 3 & $2 p_{1 / 2} 2 p_{3 / 2}^{3}$ & 2 & 9420.26 & $2.89 \times 10^{0}$ & $1.59 \times 10^{+15}$ & 1.3161 \\
\hline $2 s_{1 / 2} 2 p_{3 / 2}^{2} 3 d_{5 / 2}$ & 2 & $2 s_{1 / 2} 2 p_{3 / 2}^{3}$ & 1 & 9413.68 & $3.20 \times 10^{0}$ & $2.44 \times 10^{+15}$ & 1.317 \\
\hline $2 s_{1 / 2} 2 p_{3 / 2}^{2} 3 d_{5 / 2}$ & 3 & $2 s_{1 / 2} 2 p_{3 / 2}^{3}$ & 2 & 9412.27 & $2.45 \times 10^{0}$ & $1.35 \times 10^{+15}$ & 1.3172 \\
\hline $2 p_{1 / 2} 2 p_{3 / 2}^{2} 3 d_{5 / 2}$ & 2 & $2 p_{1 / 2} 2 p_{3 / 2}^{3}$ & 2 & 9409.66 & $2.48 \times 10^{0}$ & $1.90 \times 10^{+15}$ & 1.3176 \\
\hline $2 s_{1 / 2} 2 p_{1 / 2} 2 p_{3 / 2}^{3}$ & 2 & $2 s_{1 / 2} 2 p_{1 / 2}$ & 1 & 9378.3 & $3.02 \times 10^{0}$ & $2.33 \times 10^{+15}$ & 1.322 \\
\hline $2 s_{1 / 2} 2 p_{1 / 2} 2 p_{3 / 2}^{3}$ & 0 & $2 s_{1 / 2} 2 p_{1 / 2}$ & 1 & 9372.29 & $8.11 \times 10^{-1}$ & $3.09 \times 10^{+15}$ & 1.3228 \\
\hline $2 p_{1 / 2} 2 p_{3 / 2}^{2} 3 d_{5 / 2}$ & 1 & $2 p_{1 / 2} 2 p_{3 / 2}^{3}$ & 1 & 9365.21 & $1.04 \times 10^{0}$ & $1.33 \times 10^{+15}$ & 1.3238 \\
\hline $2 p_{3 / 2}^{3} 3 d_{5 / 2}$ & 1 & $2 p_{3 / 2}^{4}$ & 0 & 9364.03 & $2.43 \times 10^{0}$ & $3.13 \times 10^{+15}$ & 1.324 \\
\hline $2 p_{1 / 2} 3 d_{3 / 2}$ & 1 & $2 p_{3 / 2}^{4}$ & 0 & 9362.74 & $1.16 \times 10^{0}$ & $1.90 \times 10^{+15}$ & 1.3242 \\
\hline $2 p_{3 / 2} 3 d_{5 / 2}$ & 2 & $2 p_{3 / 2}^{2}$ & 2 & 9360.85 & $1.36 \times 10^{0}$ & $1.04 \times 10^{+15}$ & 1.3245 \\
\hline $2 p_{1 / 2} 2 p_{3 / 2}^{2} 3 d_{5 / 2}$ & 2 & $2 p_{1 / 2} 2 p_{3 / 2}^{3}$ & 1 & 9352.89 & $2.30 \times 10^{0}$ & $1.77 \times 10^{+15}$ & 1.3256 \\
\hline $2 s_{1 / 2} 2 p_{3 / 2}^{2} 3 d_{5 / 2}$ & 3 & $2 s_{1 / 2} 2 p_{3 / 2}^{3}$ & 2 & 9349.95 & $2.30 \times 10^{0}$ & $1.25 \times 10^{+15}$ & 1.326 \\
\hline $2 p_{3 / 2}^{3} 5 d_{5 / 2}$ & 1 & $2 p_{3 / 2}^{4}$ & 0 & 8650.1 & $1.74 \times 10^{-1}$ & $4.82 \times 10^{+14}$ & 1.4333 \\
\hline $2 p_{1 / 2} 3 p_{1 / 2}$ & 1 & $2 s_{1 / 2} 2 p_{1 / 2}$ & 1 & 8638.62 & $3.15 \times 10^{-1}$ & $5.09 \times 10^{+14}$ & 1.4352 \\
\hline $2 s_{1 / 2} 2 p_{1 / 2} 2 p_{3 / 2}^{3} 3 s_{1 / 2}$ & 2 & $2 s_{1 / 2} 2 p_{3 / 2}^{3}$ & 2 & 8597.05 & $2.40 \times 10^{-1}$ & $3.95 \times 10^{+14}$ & 1.4421 \\
\hline $2 s_{1 / 2} 2 p_{3 / 2}^{2} 3 d_{5 / 2}$ & 0 & $2 s_{1 / 2} 2 p_{3 / 2}^{3}$ & 1 & 8596.3 & $3.23 \times 10^{-1}$ & $1.21 \times 10^{+15}$ & 1.4422 \\
\hline
\end{tabular}


TABLE SIII

Forbidden transitions (E2, M1, and M2) atomic data parameters for strong lines calculated by using MR-MBPT method

\begin{tabular}{|c|c|c|c|c|c|c|c|}
\hline Upper state & $J_{\text {up }}$ & Lower state & $J_{\text {low }}$ & $\Delta E[\mathrm{eV}]$ & $g f_{i j}$ & $A_{r}\left[\mathrm{~s}^{-1}\right]$ & $\lambda[\AA]$ \\
\hline \multicolumn{8}{|c|}{ E2 transitions } \\
\hline $2 p_{1 / 2} 2 p_{3 / 2}^{2} 3 s_{1 / 2}$ & 4 & $2 p_{1 / 2}^{2} 2 p_{3 / 2}^{2}$ & 2 & 13835.45 & $5.22 \times 10^{-3}$ & $4.82 \times 10^{+12}$ & 0.896 \\
\hline $2 p_{3 / 2} 4 f_{7 / 2}$ & 4 & $2 p_{1 / 2}^{2} 2 p_{3 / 2}^{2}$ & 2 & 12457.44 & $1.01 \times 10^{-2}$ & $7.59 \times 10^{+12}$ & 0.995 \\
\hline $2 p_{3 / 2}^{3} 4 f_{7 / 2}$ & 2 & $2 p_{3 / 2}^{4}$ & 0 & 12368.94 & $6.22 \times 10^{-3}$ & $8.25 \times 10^{+12}$ & 1.002 \\
\hline $2 s_{1 / 2} 2 p_{1 / 2} 2 p_{3 / 2}^{3} 3 d_{5 / 2}$ & 3 & $2 p_{1 / 2} 2 p_{3 / 2}^{3}$ & 1 & 11247.55 & $9.70 \times 10^{-3}$ & $7.60 \times 10^{+12}$ & 1.102 \\
\hline $2 s_{1 / 2} 2 p_{3 / 2}^{2} 3 d_{5 / 2}$ & 4 & $2 p_{1 / 2}^{2} 2 p_{3 / 2}^{2}$ & 2 & 11236.6 & $1.41 \times 10^{-2}$ & $8.58 \times 10^{+12}$ & 1.103 \\
\hline $2 p_{3 / 2}^{3} 3 d_{5 / 2}$ & 4 & $2 s_{1 / 2} 2 p_{3 / 2}^{3}$ & 2 & 11233.17 & $1.48 \times 10^{-2}$ & $8.99 \times 10^{+12}$ & 1.104 \\
\hline $2 s_{1 / 2} 3 d_{5 / 2}$ & 2 & $2 p_{3 / 2}^{4}$ & 0 & 11218.25 & $1.17 \times 10^{-2}$ & $1.28 \times 10^{+13}$ & 1.105 \\
\hline $2 p_{1 / 2} 3 d_{5 / 2}$ & 3 & $2 s_{1 / 2} 2 p_{1 / 2}$ & 1 & 11207.83 & $1.17 \times 10^{-2}$ & $9.09 \times 10^{+12}$ & 1.106 \\
\hline $2 s_{1 / 2} 2 p_{1 / 2} 2 p_{3 / 2}^{3} 3 d_{5 / 2}$ & 2 & $2 p_{1 / 2} 2 p_{3 / 2}^{3}$ & 2 & 11185.31 & $1.27 \times 10^{-2}$ & $1.38 \times 10^{+13}$ & 1.108 \\
\hline $2 s_{1 / 2} 2 p_{1 / 2} 2 p_{3 / 2}^{3} 3 d_{5 / 2}$ & 3 & $2 p_{1 / 2} 2 p_{3 / 2}^{3}$ & 2 & 11171.36 & $9.63 \times 10^{-3}$ & $7.45 \times 10^{+12}$ & 1.11 \\
\hline $2 s_{1 / 2} 2 p_{3 / 2}^{2} 3 d_{5 / 2}$ & 3 & $2 p_{1 / 2}^{2} 2 p_{3 / 2}^{2}$ & 2 & 11160.99 & $1.52 \times 10^{-2}$ & $1.17 \times 10^{+13}$ & 1.111 \\
\hline $2 s_{1 / 2} 2 p_{1 / 2} 2 p_{3 / 2}^{3} 3 d_{3 / 2}$ & 2 & $2 p_{1 / 2} 2 p_{3 / 2}^{3}$ & 1 & 11160.64 & $8.07 \times 10^{-3}$ & $8.72 \times 10^{+12}$ & 1.111 \\
\hline \multicolumn{8}{|c|}{ M1 transitions } \\
\hline $2 p_{1 / 2} 2 p_{3 / 2}^{2} 5 p_{1 / 2}$ & 0 & $2 p_{1 / 2} 2 p_{3 / 2}^{3}$ & 1 & 13752.05 & $1.86 \times 10^{-6}$ & $1.53 \times 10^{+10}$ & 0.902 \\
\hline $2 p_{1 / 2} 2 p_{3 / 2}^{2} 4 p_{1 / 2}$ & 2 & $2 p_{1 / 2} 2 p_{3 / 2}^{3}$ & 2 & 12157.09 & $1.33 \times 10^{-5}$ & $1.70 \times 10^{+10}$ & 1.02 \\
\hline $2 s_{1 / 2} 2 p_{1 / 2} 2 p_{3 / 2}^{3} 4 p_{1 / 2}$ & 2 & $2 s_{1 / 2} 2 p_{1 / 2}$ & 1 & 12099.65 & $1.22 \times 10^{-5}$ & $1.55 \times 10^{+10}$ & 1.025 \\
\hline $2 s_{1 / 2} 2 p_{3 / 2}^{2} 4 p_{1 / 2}$ & 2 & $2 s_{1 / 2} 2 p_{3 / 2}^{3}$ & 2 & 12084.48 & $1.80 \times 10^{-5}$ & $2.28 \times 10^{+10}$ & 1.026 \\
\hline $2 p_{3 / 2}^{3} 3 p_{3 / 2}$ & 3 & $2 p_{1 / 2} 2 p_{3 / 2}^{3}$ & 2 & 10591.33 & $3.00 \times 10^{-5}$ & $2.09 \times 10^{+10}$ & 1.171 \\
\hline $2 s_{1 / 2} 3 p_{3 / 2}$ & 2 & $2 s_{1 / 2} 2 p_{1 / 2}$ & 1 & 10567.38 & $2.04 \times 10^{-5}$ & $1.98 \times 10^{+10}$ & 1.173 \\
\hline $2 s_{1 / 2} 2 p_{1 / 2} 2 p_{3 / 2}^{3} 3 p_{3 / 2}$ & 2 & $2 s_{1 / 2} 2 p_{3 / 2}^{3}$ & 2 & 10557.53 & $2.28 \times 10^{-5}$ & $2.21 \times 10^{+10}$ & 1.174 \\
\hline $2 s_{1 / 2} 2 p_{1 / 2} 2 p_{3 / 2}^{3} 3 p_{3 / 2}$ & 3 & $2 s_{1 / 2} 2 p_{3 / 2}^{3}$ & 2 & 10503.09 & $2.49 \times 10^{-5}$ & $1.70 \times 10^{+10}$ & 1.18 \\
\hline $2 s_{1 / 2} 2 p_{3 / 2}^{2} 3 p_{3 / 2}$ & 1 & $2 s_{1 / 2} 2 p_{3 / 2}^{3}$ & 2 & 9106.67 & $1.36 \times 10^{-5}$ & $1.63 \times 10^{+10}$ & 1.361 \\
\hline $2 p_{3 / 2}^{3} 3 p_{1 / 2}$ & 1 & $2 p_{3 / 2}^{4}$ & 0 & 8782.21 & $3.07 \times 10^{-5}$ & $3.42 \times 10^{+10}$ & 1.412 \\
\hline $2 p_{1 / 2} 2 p_{3 / 2}^{2} 3 p_{1 / 2}$ & 1 & $2 p_{1 / 2} 2 p_{3 / 2}^{3}$ & 1 & 8742.88 & $3.83 \times 10^{-5}$ & $4.24 \times 10^{+10}$ & 1.418 \\
\hline $2 p_{1 / 2} 2 p_{3 / 2}^{2} 3 p_{1 / 2}$ & 2 & $2 p_{1 / 2} 2 p_{3 / 2}^{3}$ & 2 & 8732.21 & $3.96 \times 10^{-5}$ & $2.62 \times 10^{+10}$ & 1.42 \\
\hline $2 s_{1 / 2} 2 p_{3 / 2}^{2} 3 p_{1 / 2}$ & 1 & $2 s_{1 / 2} 2 p_{3 / 2}^{3}$ & 1 & 8717.98 & $3.95 \times 10^{-5}$ & $4.34 \times 10^{+10}$ & 1.422 \\
\hline $2 s_{1 / 2} 2 p_{3 / 2}^{2} 3 p_{1 / 2}$ & 2 & $2 s_{1 / 2} 2 p_{3 / 2}^{3}$ & 2 & 8695.32 & $6.19 \times 10^{-5}$ & $4.06 \times 10^{+10}$ & 1.426 \\
\hline $2 p_{3 / 2}^{3} 3 p_{1 / 2}$ & 1 & $2 p_{3 / 2}^{4}$ & 0 & 8680.17 & $2.84 \times 10^{-5}$ & $3.10 \times 10^{+10}$ & 1.428 \\
\hline \multicolumn{8}{|c|}{ M2 transitions } \\
\hline $2 s_{1 / 2} 2 p_{3 / 2}^{2} 5 d_{5 / 2}$ & 2 & $2 s_{1 / 2} 2 p_{3 / 2}^{3}$ & 2 & 13766.32 & $1.93 \times 10^{-5}$ & $5.28 \times 10^{+10}$ & 0.901 \\
\hline $2 p_{3 / 2} 4 d_{5 / 2}$ & 4 & $2 p_{1 / 2}^{2} 2 p_{3 / 2}^{2}$ & 4 & 9397.458 & $1.68 \times 10^{-4}$ & $1.29 \times 10^{+11}$ & 1.319 \\
\hline $2 p_{3 / 2}^{3} 3 d_{5 / 2}$ & 4 & $2 p_{3 / 2}^{4}$ & 0 & 9393.341 & $1.80 \times 10^{-4}$ & $1.38 \times 10^{+11}$ & 1.32 \\
\hline $2 p_{3 / 2} 5 d_{5 / 2}$ & 4 & $2 p_{3 / 2}^{4}$ & 0 & 9393.341 & $1.88 \times 10^{-4}$ & $1.44 \times 10^{+11}$ & 1.32 \\
\hline $2 p_{1 / 2} 2 p_{3 / 2}^{2} 3 d_{5 / 2}$ & 8 & $2 p_{1 / 2} 2 p_{3 / 2}^{3}$ & 4 & 9372.913 & $2.62 \times 10^{-4}$ & $1.11 \times 10^{+11}$ & 1.323 \\
\hline $2 p_{1 / 2} 2 p_{3 / 2}^{2} 4 f_{5 / 2}$ & 8 & $2 p_{1 / 2} 2 p_{3 / 2}^{3}$ & 4 & 9372.913 & $2.75 \times 10^{-4}$ & $1.16 \times 10^{+11}$ & 1.323 \\
\hline $2 s_{1 / 2} 2 p_{3 / 2}^{2} 4 p_{1 / 2}$ & 2 & $2 s_{1 / 2} 2 p_{3 / 2}^{3}$ & 2 & 9362.741 & $1.05 \times 10^{-4}$ & $1.34 \times 10^{+11}$ & 1.324 \\
\hline $2 p_{1 / 2} 2 p_{3 / 2}^{2} 4 f_{7 / 2}$ & 2 & $2 s_{1 / 2} 2 p_{3 / 2}^{3}$ & 4 & 9351.663 & $1.09 \times 10^{-4}$ & $1.38 \times 10^{+11}$ & 1.326 \\
\hline $2 p_{3 / 2}^{3} 4 d_{5 / 2}$ & 6 & $2 s_{1 / 2} 2 p_{1 / 2}$ & 2 & 9331.514 & $1.75 \times 10^{-4}$ & $9.45 \times 10^{+10}$ & 1.329 \\
\hline $2 p_{1 / 2} 2 p_{3 / 2}^{2} 4 f_{7 / 2}$ & 8 & $2 s_{1 / 2} 2 p_{3 / 2}^{3}$ & 4 & 9313.58 & $2.74 \times 10^{-4}$ & $1.15 \times 10^{+11}$ & 1.331 \\
\hline $2 s_{1 / 2} 2 p_{1 / 2} 2 p_{3 / 2}^{3} 4 s_{1 / 2}$ & 4 & $2 s_{1 / 2} 2 p_{1 / 2}$ & 0 & 9308.233 & $1.88 \times 10^{-4}$ & $1.41 \times 10^{+11}$ & 1.332 \\
\hline
\end{tabular}

